\title{
Reduction and Optimization of Supplier Risk Indicators Based on Rough Set
}

\author{
Hongjun Guan ${ }^{\mathrm{a},{ }^{*}}$ and Aiwu Zhao ${ }^{\mathrm{b}}$ \\ ${ }^{a}$ School of Management Science and Engineering, Shandong University of Finance and Economics, Jinan Shandong, \\ 250014, China; ${ }^{b}$ School of Management, Jiangsu university, Zhenjiang Jiangsu, 212013, China
}

\begin{abstract}
The determination of supplier risk indicators is complex. Using vast data from SAP system of the enterprise, risk warning indicators can be reduced and optimized by the method of rough set. First of all, extract historical data from SAP system, and determine the discrete rules as excellent, good, moderate, and poor for each risk indicators to construct knowledge set which can be used for rough set operation. Then, using rough set theory to divide decision attribute set into equivalence classes, reduce non essential attributes, and calculate the dependence and importance degree for each essential attributes. After the normalization for all essential attributes, the reduced and optimized indicators for supplier risk evaluation system can be reached.
\end{abstract}

Keywords: Attribute reduction, discretization, optimization, rough set, supplier risk.

\section{INTRODUCTION}

How to establish an effective supplier risk evaluation indicator system gets attention by domestic and foreign experts and scholars. Dickson (1996) [1] proposed 23 indicators to evaluate supplier risk. Wang (2010) [2] constructed an evaluation indicator system consisted of 6 first level indicators and 22 two level indicators. Liu (2010) [3] put forward seven non-financial indicators which represent suppliers' interests and realization method. Zhao (2011) [4] suggested to divide supplier risk indicators into supplier core competence evaluation indicators and performance evaluation indicators, and used ANP method to calculate the ranking and weight of supplier performance evaluation indicators. At present, most supplier risk evaluation is based on the establishment of risk indicators, which is obtained by traditional questionnaire and expert scoring method. However, to reduce so large amount of indicators, experts ought to constantly revise the scoring results and adjust to the judgment matrix to satisfy the consistency check. Although this excludes non conformance during indicator establishment, because of too many subjective factors, it can not avoid the serious one sidedness of individual decision maker. Based on the confirmed basic risk indicator system of supplier risk, make full use of vast historical data from SAP system, using rough set theory to reduce and optimize risk indicators, can access to relatively actual objective risk indicators, which can reflect the supplier risk more accurately.

\section{ROUGH SET THEORY}

Rough set theory is a data analysis theory proposed by Polish mathematician professor Z. Pawlak in 1980. It can effectively analyze and treat the imprecise, inconsistent and incomplete information, so as to find connotative knowledge, and reveal potential rules [5]. The main idea of rough set is according to the given knowledge of existing problem, classify and treat the actual measured data, and divide the domain of the problem into equivalence classes, to reduce the data and derive knowledge reduction and nuclear based on the remaining of key data information. It can evaluate the dependency between data and derive classification rules of concept. Rough set divides researched domain knowledge into knowledge expression system based on the indiscernible relationship, and uses utilization, lower approximation set to approximate to the described objects, through knowledge reduction, to get the most simplified knowledge $[6,7]$. Rough set is based on knowledge and classification. However, knowledge is based on the ability to classify objects, and consists of classification mode. Different attribute knowledge description can get different classification. Classification is mainly used to produce category, these categories form knowledge module, which is the classification of class [8].

\section{REDUCTION AND OPTIMIZATION STEPS OF SUP- PLIER RISK INDICATORS BASED ON ROUGHT SET}

As a combination of qualitative analysis and quantitative tool, rough set can find rules from complicated and seemingly disordered data. At the same time, as a successful enterprise management system, SAP system provides massive basis data to find such rules. During the process of reduction and optimization of supplier risk indicators, based on the large amount of historical data in SAP system as a sample, our research use rough set theory to find rules. The specific steps are as follows:

Step 1: Set the range of each indicator according to the relationship between original data, and retrieve data from SAP database conforms to the condition, so as to establish knowledge set. 
Table 1. SAP historical data according to risk early warning indicators for suppliers of common centrifugal pump accessories.

\begin{tabular}{|c|c|c|c|c|c|c|c|c|c|c|c|c|c|c|c|c|c|c|c|c|c|c|c|c|}
\hline \multirow{2}{*}{$\begin{array}{c}\text { Supplier } \\
\text { No }\end{array}$} & \multicolumn{23}{|c|}{ Value of Risk Indicator } & \multirow[b]{2}{*}{ Result } \\
\hline & $\begin{array}{c}\text { A1 } \\
\%\end{array}$ & $\begin{array}{l}\mathrm{A2} \\
\%\end{array}$ & $\begin{array}{l}\text { A3 } \\
\%\end{array}$ & $\begin{array}{c}\text { A4 } \\
\%\end{array}$ & A5 & A6 & $\begin{array}{l}\text { A7 } \\
\%\end{array}$ & $\begin{array}{l}\text { B1 } \\
\%\end{array}$ & $\begin{array}{l}\text { B2 } \\
\%\end{array}$ & B3 & B4 & $\begin{array}{l}\text { B5 } \\
\%\end{array}$ & $\begin{array}{l}\text { B6 } \\
\%\end{array}$ & $\begin{array}{l}\text { B7 } \\
\%\end{array}$ & C1 & $\begin{array}{l}\mathrm{C} 2 \\
\%\end{array}$ & $\begin{array}{l}\mathrm{C} 3 \\
\%\end{array}$ & C4 & C5 & $\begin{array}{l}\text { D1 } \\
\%\end{array}$ & D2 & D3 & D4 & \\
\hline 1 & 0.3 & 0.4 & 92 & 100 & 2 & 1 & 98 & 0.3 & 1.5 & 1 & 3 & 10 & 15 & 0.5 & 11 & 0.6 & 0 & 1 & 2 & 95 & 5 & 1 & 95 & 1 \\
\hline 2 & 0.2 & 0.2 & 93 & 99 & 8 & 1 & 96 & 0.9 & 1.5 & 1 & 3 & 10 & 15 & 0.5 & 14 & 0.8 & 0 & 1 & 2 & 95 & 5 & 1 & 95 & 1 \\
\hline 3 & 0.2 & 0.3 & 91 & 93 & 5 & 1 & 95 & 0.2 & 1.5 & 1 & 3 & 10 & 15 & 0.5 & 15 & 0.4 & 0 & 1 & 2 & 95 & 5 & 1 & 95 & 2 \\
\hline 4 & 0.4 & 0.1 & 94 & 94 & 7 & 1 & 93 & 0.7 & 1.5 & 1 & 3 & 10 & 15 & 0.5 & 21 & 0.3 & 0 & 1 & 2 & 95 & 5 & 1 & 95 & 1 \\
\hline 5 & 0.8 & 0.9 & 88 & 89 & 11 & 1 & 87 & 1.4 & 1.5 & 1 & 3 & 10 & 15 & 0.5 & 17 & 0.1 & 0.6 & 1 & 2 & 95 & 5 & 1 & 95 & 2 \\
\hline 6 & 0.7 & 0.8 & 86 & 87 & 14 & 1 & 82 & 2.3 & 1.5 & 1 & 3 & 10 & 15 & 0.5 & 23 & 0 & 0.7 & 1 & 2 & 95 & 5 & 1 & 95 & 2 \\
\hline 7 & 1.2 & 0.6 & 85 & 88 & 12 & 1 & 89 & 3.8 & 1.5 & 1 & 3 & 10 & 15 & 0.5 & 28 & 0 & 0.6 & 1 & 2 & 95 & 5 & 1 & 95 & 2 \\
\hline 8 & 1.3 & 0.7 & 89 & 89 & 13 & 1 & 83 & 3.2 & 1.5 & 1 & 3 & 10 & 15 & 0.5 & 29 & 0 & 0.8 & 1 & 2 & 95 & 5 & 1 & 95 & 3 \\
\hline 9 & 0.9 & 1.2 & 87 & 86 & 16 & 1 & 86 & 3.7 & 1.5 & 1 & 3 & 10 & 15 & 0.5 & 22 & 0 & 0.8 & 1 & 2 & 95 & 5 & 1 & 95 & 2 \\
\hline 10 & 0.6 & 1.3 & 88 & 89 & 19 & 1 & 81 & 2.9 & 1.5 & 1 & 3 & 10 & 15 & 0.5 & 26 & 0 & 0.9 & 1 & 2 & 95 & 5 & 1 & 95 & 3 \\
\hline 11 & 1.4 & 1.2 & 85 & 85 & 18 & 1 & 86 & 1.9 & 2.5 & 1 & 3 & 10 & 15 & 0.5 & 16 & 0 & 0.7 & 1 & 2 & 95 & 5 & 1 & 95 & 2 \\
\hline 12 & 1.3 & 0.8 & 86 & 89 & 17 & 1 & 82 & 2.7 & 2.5 & 1 & 3 & 10 & 15 & 0.5 & 18 & 0 & 0.6 & 1 & 2 & 95 & 5 & 1 & 95 & 2 \\
\hline 13 & 0.8 & 0.9 & 87 & 88 & 22 & 1 & 89 & 2.9 & 2.5 & 1 & 3 & 10 & 15 & 0.5 & 19 & 0 & 0.9 & 1 & 2 & 95 & 5 & 1 & 95 & 2 \\
\hline 14 & 0.6 & 0.6 & 88 & 85 & 25 & 1 & 81 & 2.1 & 2.5 & 1 & 3 & 10 & 15 & 0.5 & 28 & 0 & 1 & 1 & 2 & 95 & 5 & 1 & 95 & 2 \\
\hline 15 & 1.1 & 0.7 & 89 & 86 & 28 & 1 & 85 & 2.3 & 2.5 & 1 & 3 & 10 & 15 & 0.5 & 12 & 0 & 1 & 1 & 2 & 95 & 5 & 1 & 95 & 2 \\
\hline 16 & 1.3 & 0.8 & 86 & 89 & 38 & 1 & 80 & 2.6 & 2.5 & 1 & 3 & 10 & 15 & 0.5 & 17 & 0 & 1 & 1 & 2 & 95 & 5 & 1 & 95 & 3 \\
\hline 17 & 0.9 & 1.4 & 89 & 87 & 21 & 1 & 88 & 2.8 & 2.5 & 1 & 3 & 10 & 15 & 0.5 & 13 & 0 & 1 & 1 & 2 & 95 & 5 & 1 & 95 & 2 \\
\hline 18 & 0.6 & 1.3 & 86 & 89 & 36 & 1 & 83 & 2.7 & 2.5 & 1 & 3 & 10 & 15 & 0.5 & 20 & 0 & 1 & 1 & 2 & 95 & 5 & 1 & 95 & 3 \\
\hline 19 & 1.4 & 1.5 & 87 & 88 & 33 & 1 & 87 & 2.4 & 2.5 & 1 & 3 & 10 & 15 & 0.5 & 24 & 0 & 1 & 1 & 2 & 95 & 5 & 1 & 95 & 2 \\
\hline 20 & 1.5 & 1.1 & 89 & 89 & 47 & 1 & 82 & 3.5 & 2.5 & 1 & 3 & 10 & 15 & 0.5 & 25 & 0.5 & 1 & 1 & 2 & 95 & 5 & 1 & 95 & 3 \\
\hline 21 & 2.6 & 3.2 & 86 & 84 & 61 & 2 & 79 & 6.3 & 6.5 & 2 & 2 & 0 & 10 & 5 & 6 & 1.2 & 1.6 & 2 & 3 & 90 & 3 & 1 & 95 & 2 \\
\hline 22 & 2.8 & 3.3 & 89 & 83 & 59 & 2 & 76 & 7.1 & 5.5 & 2 & 2 & 0 & 10 & 5 & 8 & 1.5 & 5.5 & 2 & 3 & 90 & 3 & 1 & 95 & 3 \\
\hline 23 & 3.2 & 2.7 & 82 & 84 & 69 & 2 & 78 & 5.7 & 5.5 & 2 & 2 & 0 & 10 & 5 & 3 & 1.1 & 1.5 & 2 & 3 & 90 & 3 & 1 & 95 & 3 \\
\hline 24 & 4.3 & 2.9 & 83 & 82 & 72 & 2 & 79 & 5.9 & 7.5 & 2 & 2 & 0 & 10 & 5 & 2 & 1.3 & 6 & 2 & 3 & 90 & 3 & 1 & 95 & 4 \\
\hline 25 & 6.2 & 7.8 & 79 & 79 & 66 & 2 & 81 & 10.6 & 15 & 2 & 1 & 0 & 15 & 10 & 30 & 4.5 & 6 & 2 & 3 & 90 & 1 & 1 & 95 & 4 \\
\hline
\end{tabular}

Step 2: According to evaluation results of users' preferences, form the decision table based on evaluation classification.

Step 3: Get reduction rules based on rough set theory, according to the confidence and support of rules to convert the rules into fuzzy constraint with priority, and then generate decision rules.

Step 4: Calculate the importance degree and weight for each indicator.

\section{CONSTRUCTION AND PRE-TREATMENT OF DECISION TABLE}

SAP system provides rich sample data for the comprehensive construction of indicator system. Sample data can be used to build decision table of supplier risk warning indicators, then use rough set theory to complete the reduction and optimization of each indicator. The optimized indicator system reduces the demand of information, and after repeated verification of actual data, it can be more accurately to reflect the true state of suppliers. To do that, first of all, it adopted the 23 indicators in literature [4] which was established by ANP method, and still took the common centrifugal pump parts suppliers as.

Some of the supplier risk indicator values in Table $\mathbf{1}$ are qualitative and others are quantitative. In accordance with the information reduction requirements for decision table, all of the attribute values need to be discredited. The meaning of the indicator values and their discretion rules are shown in Table 2. 
Table 2. Meaning and discretion rules of indicator values.

\begin{tabular}{|c|c|c|c|}
\hline Indicator No & Indicator Name & Grade of Discretion & Description \\
\hline A1 & Product quality complaint rate & $1-5$ & $1-[0,0.5 \%] ; 2-(0.5 \%-1 \%] ; 3-(1 \%-1.5 \%] ; 4-(1.5 \%-5 \%] ; 5$-More than $5 \%$ \\
\hline A2 & Return rate due to quality & $1-5$ & $1-[0,0.5 \%] ; 2-(0.5 \%-1 \%] ; 3-(1 \%-1.5 \%] ; 4-(1.5 \%-5 \%] ; 5$-More than $5 \%$ \\
\hline $\mathrm{A} 3$ & Product certification pass rate & $1-5$ & $\begin{array}{l}\text { 1-No less than } 90 \% \text {; 2-[85\%-90\%); } 3-[80 \%-85 \%) ; 4-[70 \%-80 \%) \text {; } 5 \text {-Less } \\
\text { than } 70 \%\end{array}$ \\
\hline A4 & $\begin{array}{l}\text { Rate of delivery in accordance } \\
\text { with order number }\end{array}$ & $1-5$ & $\begin{array}{l}\text { 1-No less than } 95 \% ; 2-[90 \%-95 \%) ; 3-[85 \%-90 \%) ; 4-[80 \%-85 \%) ; 5 \text {-Less } \\
\text { than } 80 \%\end{array}$ \\
\hline A5 & Ranking of performance & $1-5$ & 1-Top 10; 2-(10-20]; 3-(20-50]; 4-(50, 80]; 5-After 80 \\
\hline A6 & Product grade & $1-4$ & 1-Excellent; 2-Good; 3-Moderate: 4-Other \\
\hline A7 & $\begin{array}{l}\text { Reached rate of product } \\
\text { standard life }\end{array}$ & $1-5$ & 1-No less than $90 \% ; 2-[85 \%-90 \%) ; 3-[80 \%-85 \%) ; 4$-Less than $80 \%$ \\
\hline B1 & Price parity & $1-5$ & $\begin{array}{l}\text { Higher than average: } 1 \text {-No more than } 0.5 \% ; 2-(0.5 \%-1 \%] ; 3-(1 \%-5 \%] ; 4- \\
\qquad(5 \%-10 \%] ; 5-\text { More than } 10 \%\end{array}$ \\
\hline B2 & Price discount & $1-3$ & 1-No less than $10 \%$; 2-[5\%-10\%); 3-[1\%-5\%); 4-Less than $1 \%$ \\
\hline B3 & Freight fee & $1-3$ & 1-Seller; 2-Arrive station; 3-Buyer \\
\hline B4 & Terms of payment & $1-3$ & 1-Installment; 2-Delivery pay; 3-Prepay \\
\hline B5 & Prepayment ratio of the order & $1-5$ & $1-[0,5 \%] ; 2-(5 \%-10 \%] ; 3-(10 \%-15 \%] ; 4-(15 \%-20 \%] ; 5$-More than $20 \%$ \\
\hline B6 & $\begin{array}{l}\text { Payment ratio for quality } \\
\text { warranty }\end{array}$ & $1-5$ & $1-[0,5 \%] ; 2-(5 \%-10 \%] ; 3-(10 \%-15 \%] ; 4-(15 \%-20 \%] ; 5$-More than $20 \%$ \\
\hline B7 & Financial fee & $1-5$ & $1-[0,1 \%] ; 2-(1 \%-3 \%] ; 3-(3 \%-5 \%] ; 4-(5 \%-10 \%] ; 5-M o r e$ than $10 \%$ \\
\hline $\mathrm{C} 1$ & Ranking of arrival time & $1-5$ & 1-Top 10;2-(10-30];3-(30-50];4-(50, 80];5-After 80 \\
\hline $\mathrm{C} 2$ & Ratio of delayed delivery & $1-5$ & $1-[0,1 \%] ; 2-(1 \%-3 \%] ; 3-(3 \%-5 \%] ; 4-(5 \%-10 \%] ; 5-$ More than $10 \%$ \\
\hline $\mathrm{C} 3$ & Breach of contract & $1-5$ & $1-[0,0.5 \%] ; 2-(0.5 \%-1 \%] ; 3-(1 \%-5 \%] ; 4-(5 \%-10 \%] ; 5$-More than $10 \%$ \\
\hline $\mathrm{C} 4$ & Delivery method & $1-4$ & 1-Motor transport; 2-Rail transport; 3-Pipeline transport; 4-Other \\
\hline $\mathrm{C} 5$ & Producing area and the distance & $1-4$ & 1-City; 2-Province; 3-Domestic; 4-Oversea \\
\hline D1 & Service prompt rate after sale & $1-5$ & $\begin{array}{l}\text { 1-No less than } 95 \% ; 2-[90 \%-95 \%) ; 3-[85 \%-90 \%) ; 4-[80 \%-85 \%) ; 5 \text {-Less } \\
\text { than } 80 \%\end{array}$ \\
\hline D2 & Guarantee period & $1-4$ & 1-No less than 5 years;2-[3-5);3-[1-3);4-In one year \\
\hline D3 & $\begin{array}{l}\text { Communication skills of busi- } \\
\text { ness man }\end{array}$ & $1-4$ & 1-Excellent; 2-Good; 3-Moderate; 4-Other \\
\hline D4 & Regular visit & $1-5$ & $\begin{array}{l}\text { 1-No less than } 95 \% ; 2-[90 \%-95 \%) ; 3-[85 \%-90 \%) ; 4-[80 \%-85 \%) ; 5 \text {-Less } \\
\text { than } 80 \%\end{array}$ \\
\hline
\end{tabular}

According to the rules of discretion in Table 2, adding evaluation result of excellent, good, moderate, poor (R1, R2, $\mathrm{R} 3$, R4), the statistical data of decision table in Table $\mathbf{1}$ is simplified as shown in Table $\mathbf{3}$.

\section{REDUCTION AND OPTIMIZATION OF SUPPLIER RISK INDICATORS}

\subsection{Divide Equivalence Classes for Each Risk Indicator}

According to decision Table 3, the equivalence classes are divided as follows:
$\mathrm{U} / \mathrm{C}=\{\{\mathrm{u} 1\},\{\mathrm{u} 2\},\{\mathrm{u} 3\},\{\mathrm{u} 4\},\{\mathrm{u} 5\},\{\mathrm{u} 6\},\{\mathrm{u} 7\},\{\mathrm{u} 8\}$, $\{\mathrm{u} 9\},\{\mathrm{u} 10\},\{\mathrm{u} 11\},\{\mathrm{u} 12\},\{\mathrm{u} 13\},\{\mathrm{u} 14\},\{\mathrm{u} 15\},\{\mathrm{u} 16\}$, $\{u 17\},\{u 18\},\{u 19\},\{u 20\},\{u 21\},\{u 22\},\{u 23\},\{u 24\}$, $\{\mathrm{u} 25\}\}$

$\mathrm{U} / \mathrm{D}=\{\{\mathrm{u} 1, \mathrm{u} 2, \mathrm{u} 4\},\{\mathrm{u} 3, \mathrm{u} 5, \mathrm{u} 6, \mathrm{u} 7, \mathrm{u} 9, \mathrm{u} 11, \mathrm{u} 12, \mathrm{u} 13$, u14, u15, u17, u19, u21\}, \{u8, u10, u16, u18, u22, u23, $\mathrm{u} 20\},\{\mathrm{u} 24, \mathrm{u} 25\}\}$

$\mathrm{U} /\{\mathrm{C}-\{\mathrm{A} 1\}\}=\{\{\mathrm{u} 1\},\{\mathrm{u} 2\},\{\mathrm{u} 3\},\{\mathrm{u} 4\},\{\mathrm{u} 5, \mathrm{u} 7\},\{\mathrm{u} 6$, $\mathrm{u} 8\},\{\mathrm{u} 9, \mathrm{u} 11\},\{\mathrm{u} 10\},\{\mathrm{u} 12\},\{\mathrm{u} 13, \mathrm{u} 15\},\{\mathrm{u} 14, \mathrm{u} 16\},\{\mathrm{u} 17$, u19\}, $\{\mathrm{u} 18, \mathrm{u} 20\},\{\mathrm{u} 21\},\{\mathrm{u} 22\},\{\mathrm{u} 23\},\{\mathrm{u} 24\},\{\mathrm{u} 25\}\}$ 
Table 3. Simplified decision table.

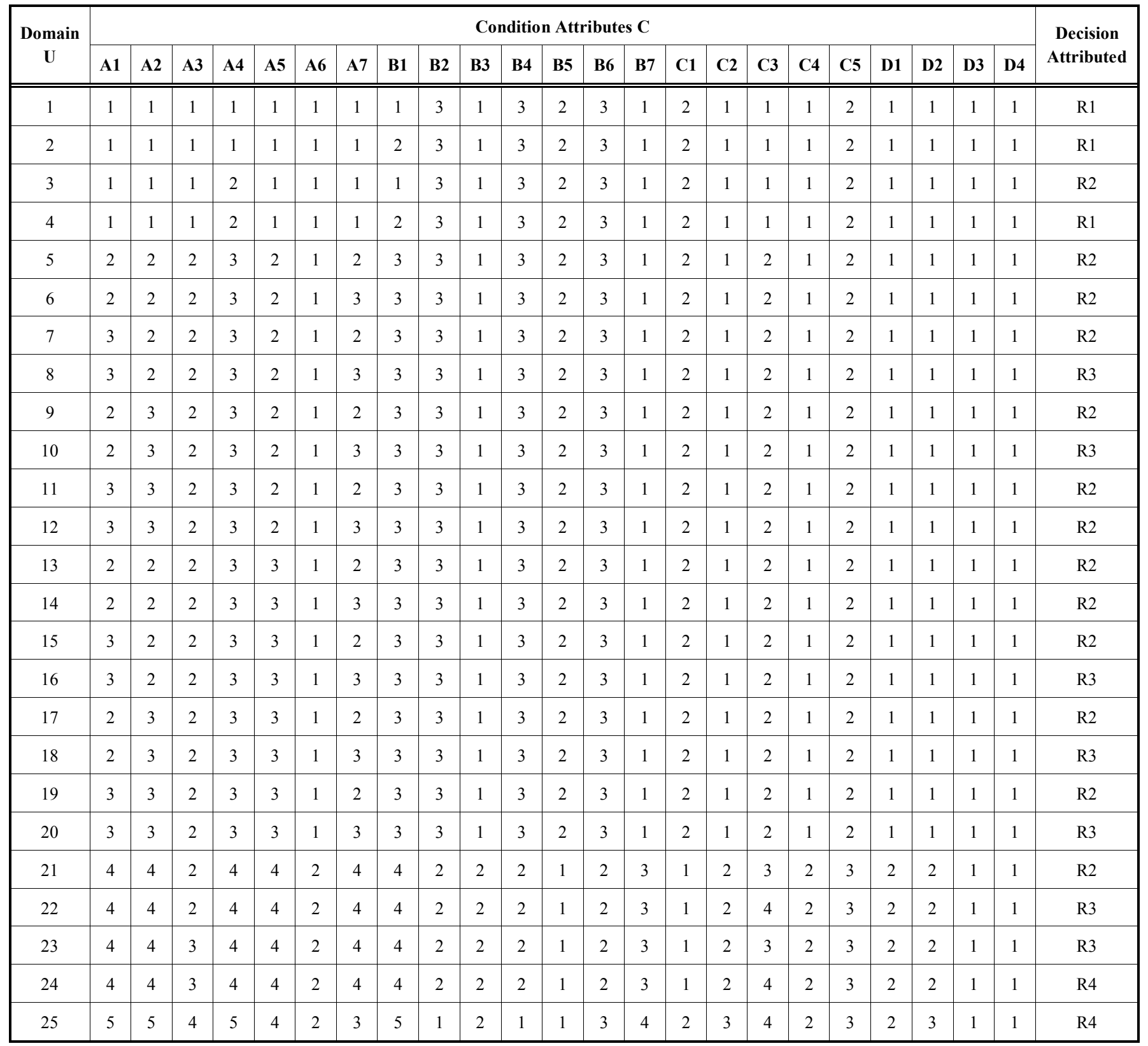

$\mathrm{U} /\{\mathrm{C}-\{\mathrm{A} 2\}\}=\{\{\mathrm{u} 1\},\{\mathrm{u} 2\},\{\mathrm{u} 3\},\{\mathrm{u} 4\},\{\mathrm{u} 5, \mathrm{u} 9\},\{\mathrm{u} 6$, $\mathrm{u} 10\},\{\mathrm{u} 7, \mathrm{u} 11\},\{\mathrm{u} 8, \mathrm{u} 12\},\{\mathrm{u} 13, \mathrm{u} 17\},\{\mathrm{u} 14, \mathrm{u} 18\},\{\mathrm{u} 15$, $\mathrm{u} 19\},\{\mathrm{u} 16, \mathrm{u} 20\},\{\mathrm{u} 21\},\{\mathrm{u} 22\},\{\mathrm{u} 23\},\{\mathrm{u} 24\},\{\mathrm{u} 25\}\}$

$\mathrm{U} /\{\mathrm{C}-\{\mathrm{A} 3\}\}=\{\{\mathrm{u} 1\},\{\mathrm{u} 2\},\{\mathrm{u} 3\},\{\mathrm{u} 4\},\{\mathrm{u} 5\},\{\mathrm{u} 6\}$, $\{u 7\},\{u 8\},\{u 9\},\{u 10\},\{u 11\},\{u 12\},\{u 13\},\{u 14\}$, $\{\mathrm{u} 15\},\{\mathrm{u} 16\},\{\mathrm{u} 17\},\{\mathrm{u} 18\},\{\mathrm{u} 19\},\{\mathrm{u} 20\},\{\mathrm{u} 21, \mathrm{u} 23\}$, $\{\mathrm{u} 22, \mathrm{u} 24\},\{\mathrm{u} 25\}\}$

$\mathrm{U} /\{\mathrm{C}-\{\mathrm{A} 4\}\}=\{\{\mathrm{u} 1, \mathrm{u} 3\},\{\mathrm{u} 2, \mathrm{u} 4\},\{\mathrm{u} 5\},\{\mathrm{u} 6\},\{\mathrm{u} 7\}$, $\{u 8\},\{u 9\},\{u 10\},\{u 11\},\{u 12\},\{u 13\},\{u 14\},\{u 15\}$, $\{\mathrm{u} 16\},\{\mathrm{u} 17\},\{\mathrm{u} 18\},\{\mathrm{u} 19\},\{\mathrm{u} 20\},\{\mathrm{u} 21\},\{\mathrm{u} 22\},\{\mathrm{u} 23\}$, $\{\mathrm{u} 24\},\{\mathrm{u} 25\}\}$

$\mathrm{U} /\{\mathrm{C}-\{\mathrm{A} 5\}\}=\{\{\mathrm{u} 1\},\{\mathrm{u} 2\},\{\mathrm{u} 3\},\{\mathrm{u} 4\},\{\mathrm{u} 5, \mathrm{u} 13\},\{\mathrm{u} 6$, $\mathrm{u} 14\},\{\mathrm{u} 7, \mathrm{u} 15\},\{\mathrm{u} 8, \mathrm{u} 16\},\{\mathrm{u} 9, \mathrm{u} 17\},\{\mathrm{u} 10, \mathrm{u} 18\},\{\mathrm{u} 11$, $\mathrm{u} 19\},\{\mathrm{u} 12, \mathrm{u} 20\},\{\mathrm{u} 21\},\{\mathrm{u} 22\},\{\mathrm{u} 23\},\{\mathrm{u} 24\},\{\mathrm{u} 25\}\}$

$\mathrm{U} /\{\mathrm{C}-\{\mathrm{A} 6\}\}=\mathrm{U} /\{\mathrm{C}-\{\mathrm{A} 5\}\}$
$\mathrm{U} /\{\mathrm{C}-\{\mathrm{A} 7\}\}=\{\{\mathrm{u} 1\},\{\mathrm{u} 2\},\{\mathrm{u} 3\},\{\mathrm{u} 4\},\{\mathrm{u} 5, \mathrm{u} 6\},\{\mathrm{u} 7$, $\mathrm{u} 8\},\{\mathrm{u} 9, \mathrm{u} 10\},\{\mathrm{u} 11, \mathrm{u} 12\},\{\mathrm{u} 13, \mathrm{u} 14\},\{\mathrm{u} 15, \mathrm{u} 16\},\{\mathrm{u} 17$, $\mathrm{u} 18\},\{\mathrm{u} 19, \mathrm{u} 20\},\{\mathrm{u} 21\},\{\mathrm{u} 22\},\{\mathrm{u} 23\},\{\mathrm{u} 24\},\{\mathrm{u} 25\}\}$

$\mathrm{U} /\{\mathrm{C}-\{\mathrm{B} 1\}\}=\{\{\mathrm{u} 1, \mathrm{u} 2\},\{\mathrm{u} 3, \mathrm{u} 4\},\{\mathrm{u} 5\},\{\mathrm{u} 6\},\{\mathrm{u} 7\}$, $\{\mathrm{u} 8\},\{\mathrm{u} 9\},\{\mathrm{u} 10\},\{\mathrm{u} 11\},\{\mathrm{u} 12\},\{\mathrm{u} 13\},\{\mathrm{u} 14\},\{\mathrm{u} 15\}$, $\{\mathrm{u} 16\},\{\mathrm{u} 17\},\{\mathrm{u} 18\},\{\mathrm{u} 19\},\{\mathrm{u} 20\},\{\mathrm{u} 21\},\{\mathrm{u} 22\},\{\mathrm{u} 23\}$, $\{\mathrm{u} 24\},\{\mathrm{u} 25\}\}$
$\mathrm{U} /\{\mathrm{C}-\{\mathrm{B} 2\}\}=\mathrm{U} /\{\mathrm{C}-\{\mathrm{A} 4\}\}$
$\mathrm{U} /\{\mathrm{C}-\{\mathrm{B} 3\}\}=\mathrm{U} / \mathrm{C}$
$\mathrm{U} /\{\mathrm{C}-\{\mathrm{B} 4\}\}=\mathrm{U} / \mathrm{C}$
$\mathrm{U} /\{\mathrm{C}-\{\mathrm{B} 5\}\}=\mathrm{U} / \mathrm{C}$
$\mathrm{U} /\{\mathrm{C}-\{\mathrm{B} 6\}\}=\mathrm{U} / \mathrm{C}$
$\mathrm{U} /\{\mathrm{C}-\{\mathrm{B} 7\}\}=\mathrm{U} / \mathrm{C}$ 
$\mathrm{U} /\{\mathrm{C}-\{\mathrm{C} 1\}\}=\mathrm{U} / \mathrm{C}$

$\mathrm{U} /\{\mathrm{C}-\{\mathrm{C} 2\}\}=\mathrm{U} / \mathrm{C}$

$\mathrm{U} /\{\mathrm{C}-\{\mathrm{C} 3\}\}=\{\{\mathrm{u} 1\},\{\mathrm{u} 2\},\{\mathrm{u} 3\},\{\mathrm{u} 4\},\{\mathrm{u} 5\},\{\mathrm{u} 6\},\{\mathrm{u} 7\}$, $\{\mathrm{u} 8\},\{\mathrm{u} 9\},\{\mathrm{u} 10\},\{\mathrm{u} 11\},\{\mathrm{u} 12\},\{\mathrm{u} 13\},\{\mathrm{u} 14\},\{\mathrm{u} 15\}$, $\{u 16\},\{u 17\},\{u 18\},\{u 19\},\{u 20\},\{u 21, u 22\},\{u 23, u 24\}$, $\{\mathrm{u} 25\}$ \}

$$
\begin{aligned}
& \mathrm{U} /\{\mathrm{C}-\{\mathrm{C} 4\}\}=\mathrm{U} / \mathrm{C} \\
& \mathrm{U} /\{\mathrm{C}-\{\mathrm{C} 5\}\}=\mathrm{U} / \mathrm{C} \\
& \mathrm{U} /\{\mathrm{C}-\{\mathrm{D} 1\}\}=\mathrm{U} / \mathrm{C} \\
& \mathrm{U} /\{\mathrm{C}-\{\mathrm{D} 2\}\}=\mathrm{U} / \mathrm{C} \\
& \mathrm{U} /\{\mathrm{C}-\{\mathrm{D} 3\}\}=\mathrm{U} / \mathrm{C} \\
& \mathrm{U} /\{\mathrm{C}-\{\mathrm{D} 4\}\}=\mathrm{U} / \mathrm{C}
\end{aligned}
$$

\subsection{Calculate Positive Domain and Obtain the Impor- tance Degree of Each Indicator}

According to rough set theory and the divided equivalence classes above, calculate positive domain and the importance degree of each indicator as follows:

$\operatorname{pos} C(D)=\{u 1, u 2, u 3, u 4, u 5, u 6, u 7, u 8, u 9, u 10, u 11$, u12, u13, u14, u15, u16, u17, u18, u19, u20, u21, u22, u23, u24, u25\}

$\operatorname{pos} C-\{A 1\}(D)=\{u 1, u 2, u 3, u 4, u 5, u 7, u 9, u 10, u 11$, u12, u13, u15, u17, u18, u19, u20, u21, u22, u23, u24, $\mathrm{u} 25)\} \neq \operatorname{pos}(\mathrm{D}), \mathrm{A} 1$ is an essential condition, its importance degree: $25 / 25-21 / 25=4 / 25$

$\operatorname{pos} C-\{A 2\}(D)=\{u 1, u 2, u 3, u 4, u 5, u 7, u 9, u 11, u 13$,

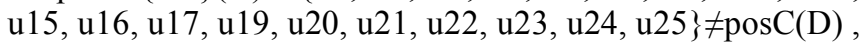
A2 is an essential condition, its importance degree: $25 / 25$ $19 / 25=6 / 25$

$\operatorname{pos} C-\{A 3\}(D)=\{u 1, u 2, u 3, u 4, u 5, u 6, u 7, u 8, u 9, u 10$, u11, u12, u13, u14, u15, u16, u17, u18, u19, u20, $\mathrm{u} 25\} \neq \operatorname{pos} C(\mathrm{D}), \mathrm{A} 3$ is an essential condition, its importance degree: $25 / 25-21 / 25=4 / 25$

$\operatorname{pos} C-\{A 4\}(D)=\{u 2, u 4, u 5, u 6, u 7, u 8, u 9, u 10, u 11$, u12, u13, u14, u15, u16, u17, u18, u19, u20, u21, u22, u23, $\mathrm{u} 24, \mathrm{u} 25\} \neq \operatorname{posC}(\mathrm{D}), \mathrm{A} 4$ is an essential condition, its importance degree: $25 / 25-23 / 25=2 / 25$

$\operatorname{pos} C-\{A 5\}(D)=\{u 1, u 2, u 3, u 4, u 5, u 6, u 7, u 8, u 9, u 10$, u11, u13, u14, u15, u16, u17, u18, u19, u21, u22, u23, u24, $\mathrm{u} 25\} \neq \operatorname{pos} C(\mathrm{D}), \mathrm{A} 5$ is an essential condition, its importance degree: $25 / 25-23 / 25=2 / 25$

$\operatorname{pos} C-\{\mathrm{A} 6\}(\mathrm{D})=\operatorname{pos} \mathrm{C}-\{\mathrm{A} 5\}(\mathrm{D}), \mathrm{A} 6$ is an essential condition, its importance degree: $2 / 25$

$\operatorname{posC}-\{\mathrm{A} 7\}(\mathrm{D})=$

\{u1, u2, u3, u4, u5, u6, u11, u12, u13, u14, u21, u22, $\mathrm{u} 23, \mathrm{u} 24, \mathrm{u} 25\} \neq \operatorname{pos} C(\mathrm{D}), \mathrm{A} 7$ is an essential condition, its importance degree: $25 / 25-15 / 25=10 / 25$

$\operatorname{pos} \mathrm{C}-\{\mathrm{B} 1\}(\mathrm{D})=$

$\{\mathrm{u} 1, \mathrm{u} 2, \mathrm{u} 5, \mathrm{u} 6, \mathrm{u} 7, \mathrm{u} 8, \mathrm{u} 9, \mathrm{u} 10, \mathrm{u} 11, \mathrm{u} 12, \mathrm{u13}, \mathrm{u14}, \mathrm{u15}$, u16, u17, u18, u19, u20, u21, u22, u23, u24, u25\}

$\neq \operatorname{posC}(\mathrm{D}), \mathrm{B} 1$ is an essential condition, its importance degree: $25 / 25-23 / 25=2 / 25$ $\operatorname{pos} C-\{\mathrm{B} 2\}(\mathrm{D})=\operatorname{pos} \mathrm{C}-\{\mathrm{A} 4\}(\mathrm{D}), \mathrm{B} 2$ is an essential condition, its importance degree: $2 / 25$

$\operatorname{pos} C-\{C 3\}(D)=\{u 1, u 2, u 3, u 4, u 5, u 6, u 7, u 8, u 9, u 10$, u11, u12, u13, u14, u15, u16, u17, u18, u19, u20, u23, u24, $\mathrm{u} 25\} \neq \operatorname{posC}(\mathrm{D}), \mathrm{C} 3$ is an essential condition, its importance degree: $25 / 25-23 / 25=2 / 25$

The other indicators are unessential conditions and can be reduced.

\subsection{Normalization and Weight of Indicators}

Within Normalized each essential indicator, the weight of reduced and optimized risk warning indicators are shown in Table 4 .

Table 4. Weight table of reduced and optimized early warning indicators by rough set.

\begin{tabular}{|c|c|c|}
\hline Indicator No & Indicator Name & Weight \\
\hline \hline A1 & Product quality complaint rate & 0.058824 \\
\hline A2 & Return rate due to quality & 0.176471 \\
\hline A3 & Product certification pass rate & 0.117647 \\
\hline A4 & $\begin{array}{c}\text { Rate of delivery in accordance with } \\
\text { order number }\end{array}$ & 0.058824 \\
\hline A5 & Ranking of performance & 0.058824 \\
\hline A6 & Product grade & 0.058824 \\
\hline A7 & Reached rate of product standard life & 0.294118 \\
\hline B1 & Price parity & 0.058824 \\
\hline B2 & Price discount & 0.058824 \\
\hline C3 & Breach of contract & 0.058824 \\
\hline
\end{tabular}

\section{SUMMARY AND CONCLUSION}

Considering the influence of subjective factors during the construction of indicator system using traditional method, based on initial determined indicators derived from traditional experience, combined with extracted historical data in enterprises' SAP system, this paper used rough set theory to reduce and optimize the original risk indicator system and lower the system dimension. Then it recalculated their weights and optimized the risk evaluation rules. Because of the using of newly real data from suppliers' business, this method can establish risk early warning indicator system for each material catalog dynamically and in real time. Indicator system which is optimized and validated can reflect the real state of suppliers more accurately with less information requirement.

\section{CONFLICT OF INTEREST}

The authors confirm that this article content has no conflict of interest.

\section{ACKNOWLEDGEMENTS}

This work is supported by the National Natural Science Foundation of China (No.71171099, 71471076, 71411170250), 
the Fund of the Ministry of Education of Humanities and Social Sciences (No.14YJAZH025), China Postdoctoral Science Foundation (No.2013M530862, 2014M551525), the Natural Science Foundation of Shandong Province (No.ZR2013GM003), and the Jiangsu Postdoctoral Research Grants Program (No.1302056C). The authors are grateful to the anonymous referee for carefully verifying the details and for providing helpful comments that improved this paper.

\section{ABOUT THE AUTHORS}

Hongjun Guan is the professor at Shandong University of Finance and Economics, the deputy director of Computer Information Application Research Institute, Doctor of Engineering, Post-doctor at Central University of Finance and Economics, Faculty of Electronic Commerce. His main research interests include enterprise informatization, ecommerce technology, decision-making science and technology, safety science and technology. In recent years, he took responsibility of 6 provincial level projects, took part in 5 provincial level projects, received 2 provincial rewards, and published more than 30 papers.

Aiwu Zhao holds a $\mathrm{PhD}$ from Wuhan University of Technology, Faculty of Safety Science and Technology, Doctor of Engineering, Post-doctor at Jiangsu University, Management Science and Engineering in Management Institute, Director in Jinan Xinzhongyuan Software Co., Ltd. Her main research interests lie in the area of enterprise informatization, computing experiment method of social science, decision-making technology, safety science technology and method. She took responsibility in 2 provincial level projects, took part in 4 provincial level projects and 2 national level projects, received 1 provincial reward, and published more than 10 papers.

\section{REFERENCES}

[1] E.J. Wilson, “The relative importance of suppliers selection criteria: a review and update," International Journal of Purchasing and Materials Management, vol. 30, no. 3, pp. 35-41, 1994.

[2] B. Wang "Suppliers evaluation and selection research based on risk analysis," Productivity Research, no. 8, pp. 99-101, 2010.

[3] L. Li. "Empirical study on importance degree of non-financial indicators based on suppliers", Journal of Shijiazhuang Tiedao University (Social Science), vol. 8, no. 4, pp. 30-35, 2010.

[4] A. Zhao, H. Guan, and G. Shi, "Suppliers risk evaluation indicators based on ANP," Statistics and Decision-making, vol. 4, no. 376, pp. 177-179, 2013.

[5] Z. Pawlak, "Rough sets", Communications of ACM, vol. 38, no. 11, pp. 89- 95, 1995.

[6] W. Ziarko. "Variable precision rough sets model", Journal of Computer and Systems Science, vol. 46, no. 1, pp. 39-59, 1993.

[7] S. Li, and F. Hu, "An exploration of green evaluation of supplier based on fuzzy rough set", The Theory and Practice of Finance and Economics, vol. 27, no. 142, pp. 97-100, 2006.

[8] Q. Shen, and X. Han, “Comparative study of fuzzy sets, rough sets and vague sets", Computer Engineering and Application, vol. 43, no. 4, pp. 36-39, 2007.

Received: September 16, 2014

Revised: December 23, 2014

Accepted: December 31, 2014

(C) Guan and Zhao; Licensee Bentham Open.

This is an open access article licensed under the terms of the Creative Commons Attribution Non-Commercial License (http://creativecommons.org/licenses/by-nc/3.0/) which permits unrestricted, non-commercial use, distribution and reproduction in any medium, provided the work is properly cited. 